ARTICLE

Received 19 Jan 2014 | Accepted 15 Apr 2014 | Published 14 May $2014 \quad$ DOl: 10.1038/ncomms4895

\title{
Coherent properties of single rare-earth spin qubits
}

P. Siyushev ${ }^{1, \star}$, K. Xia ${ }^{1}{ }^{1 \star}$, R. Reuter ${ }^{1}$, M. Jamali ${ }^{1}$, N. Zhao ${ }^{2}$, N. Yang ${ }^{3}$, C. Duan ${ }^{4}$, N. Kukharchyk ${ }^{5}$, A.D. Wieck ${ }^{5}$, R. Kolesov ${ }^{1} \&$ J. Wrachtrup ${ }^{1}$

Rare-earth-doped crystals are excellent hardware for quantum storage of photons. Additional functionality of these materials is added by their waveguiding properties allowing for on-chip photonic networks. However, detection and coherent properties of rare-earth single-spin qubits have not been demonstrated so far. Here we present experimental results on high-fidelity optical initialization, effcient coherent manipulation and optical readout of a single-electron spin of $\mathrm{Ce}^{3+}$ ion in a yttrium aluminium garnet crystal. Under dynamic decoupling, spin coherence lifetime reaches $T_{2}=2 \mathrm{~ms}$ and is almost limited by the measured spin-lattice relaxation time $T_{1}=4.5 \mathrm{~ms}$. Strong hyperfine coupling to aluminium nuclear spins suggests that cerium electron spins can be exploited as an interface between photons and long-lived nuclear spin memory. Combined with high brightness of $\mathrm{Ce}^{3+}$ emission and a possibility of creating photonic circuits out of the host material, this makes cerium spins an interesting option for integrated quantum photonics.

\footnotetext{
13. Physikalisches Institut, Universität Stuttgart and Stuttgart Research Center of Photonic Engineering (SCoPE), Pfaffenwaldring 57, Stuttgart D-70569, Germany. ${ }^{2}$ Beijing Computational Science Research Center, Beijing 100084, China. ${ }^{3}$ Institute of Applied Physics and Computational Mathematics, PO Box 8009 (28), Beijing 100088, China. ${ }^{4}$ Hefei National Laboratory for Physics Sciences at Microscale, Department of Physics, University of Science and Technology of China, Hefei 230026, China. ${ }^{5}$ Ruhr-Universität Bochum, Universitätsstraß e 150 Gebäude NB, Bochum D-44780, Germany. ${ }^{\star}$ These authors contributed equally to this work. Correspondence and requests for materials should be addressed to N.Z. (email: nzhao@csrc.ac.cn) or to R.K. (email: r.kolesov@physik.uni-stuttgart.de).
} 
T he outstandingly long optical coherence times of rareearth-based crystals result in efficient memories for quantum states of single photons ${ }^{1}$ and of entangled photon pairs ${ }^{2,3}$. Even longer coherence storage times can be achieved in nuclear spin memories ${ }^{4-6}$. In these quantum memories, the quantum state of a photon is stored in an electron or nuclear spin wave created in an ensemble of spins. This type of memory is suitable for storage at a single photon $\operatorname{level}^{7}$ and, therefore, is an essential ingredient of quantum repeaters ${ }^{8}$ and quantum-computing protocols based on linear optics 9 . However, ensemble-based rare-earth quantum computer designs lack certain functionalities such as error correction that are essential for scalability ${ }^{10}$. This problem can be resolved by addressing single rare-earth spins individually. In the last 2 years, detection of several rare-earth species at a single ion level was demonstrated $^{11-14}$, although no long-lasting spin coherence for single ions was demonstrated so far. In this Article, we for the first time demonstrate optically addressable rare-earth single-spin qubit in an oxide crystal, namely, cerium ion in yttrium aluminium garnet (YAG), which can be optically initialized, coherently manipulated with microwaves and read out optically.

\section{Results}

Optical isolation of a single $\mathrm{Ce}^{3+}$ in YAG. Experimentally, individual cerium ions were identified in an ultra-pure YAG crystal using confocal microscopy ${ }^{13}$ at $T \approx 3.5 \mathrm{~K}$ (see Methods section and Supplementary Fig. 1). In order to improve the fluorescence collection efficiency of the microscope, a solid immersion lens (SIL) was fabricated on the surface of the sample by focused ion beam (FIB) milling. Cerium ions were excited by a frequency-doubled femtosecond Ti:sapphire laser at $460 \mathrm{~nm}$ matching the wavelength of the phonon-assisted absorption band of $\mathrm{Ce}^{3+}$ in YAG. Their emission associated with the $5 d \rightarrow 4 f$ (see Fig. 1a) transition was detected in a 475-630-nm spectral window. The image of individual cerium ions under the SIL is shown in Fig. 1b. The single nature of the photon emitter was confirmed with photon antibunching measurements as indicated in Fig. 1c. The detected emission of an individual cerium ion amounts to $60-70 \times 10^{3}$ photons $\mathrm{s}^{-1}$. The emission spectrum of the ions is indicated in Fig. 1d, showing broadband phononassisted emission and a sharp zero-phonon line at $489 \mathrm{~nm}$ (see Fig. 1e) characteristic for cerium ions ${ }^{15}$. Cerium fluorescent centres can be created in YAG crystal by ion implantation (see Fig. If and Methods section for the details on implantation). The yield of fluorescent cerium ions was estimated to be at least $8 \%$. It is believed that the yield can be significantly improved by thermal annealing in a reducing atmosphere ${ }^{16}$.

Initialization of the spin state. The electronic level structure of cerium substitutional impurities in ceramic crystals is shown in Fig. 1a. The strongest transition between the ground $4 f(1)$ and the optically excited $5 d(1)$ spin doublets under $\sigma^{+}$circularly polarized excitation is the spin flip transition $|4 f(1) \downarrow\rangle \rightarrow$ $|4 d(1) \uparrow\rangle$. The other three transitions are almost three orders of magnitude weaker ${ }^{13}$. However, decay back to the two ground states is equally probable. Repeated excitation results in efficient pumping of the ion into spin-up 'dark' state, thereby initializing the cerium spin.

To further reveal the pumping dynamics of the cerium spin, a magnetic field of $490 \mathrm{G}$ parallel to the excitation beam was applied to the sample, the excitation laser was chopped such that the cerium centre is excited with a train of pulses and the time a

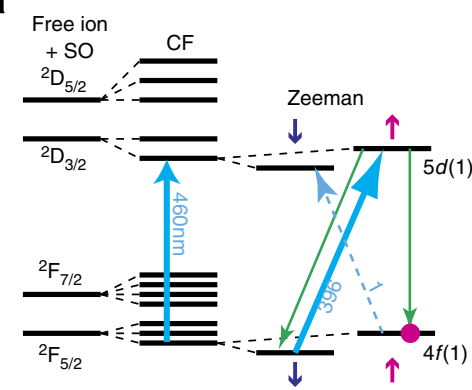

b

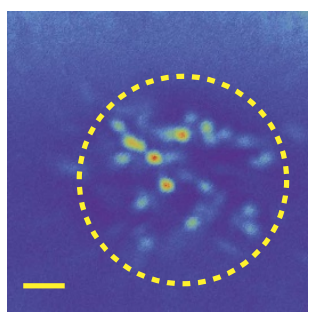

C

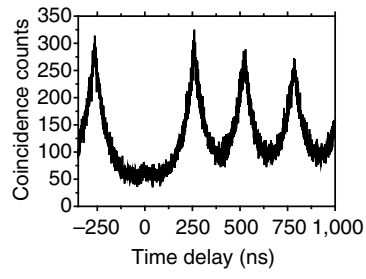

f

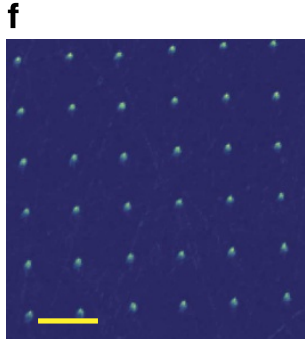

Figure 1 | General properties of a single $\mathbf{C e}^{\mathbf{3}+}$ ion in YAG. (a) Electronic level structure of $\mathrm{Ce}^{3+}$ ion in YAG crystal. Blue arrows pointing upwards indicate electronic transitions under laser excitation, while green arrows pointing downwards represent cerium fluorescence. Relative strengths of optical transitions between the lowest $4 f$ and the lowest $5 d$ spin doublets (396:1) are shown for circularly polarized excitation light propagating along the $y$ axis of the local $\mathrm{Ce}^{3+}$ symmetry frame. External magnetic field along the $y$ direction splits the spin doublets. Spin-up and spin-down states are indicated by short up- and down-arrows next to the corresponding energy level. (b) Confocal scan of individual cerium centres under the SIL. The scale bar is $2 \mu \mathrm{m}$. The dashed circle indicates boundary of the SIL. (c) Photon-correlation signal taken on a single $\mathrm{Ce}^{3+}$ centre under pulsed laser excitation (see Methods section for details on antibunching measurements). The absence of the fluorescence peak at zero delay indicates single nature of the photon emitter. (d) Typical spectrum of a single cerium ion at $3.5 \mathrm{~K}$. (e) Higher-resolution spectrum of $\mathrm{Ce}^{3+}$ in the vicinity of its zero-phonon line (ZPL). The positions of ZPLs for distinct cerium centres are slightly different depending on the local environment of each centre. (f) Focused beam of cerium ions ( 300 keV) was used to create a grid of $\mathrm{Ce}^{3+}$-doped spots in YAG single crystal. The scale bar is $10 \mu \mathrm{m}$. 
a

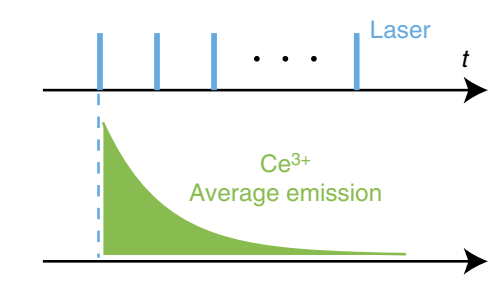

b

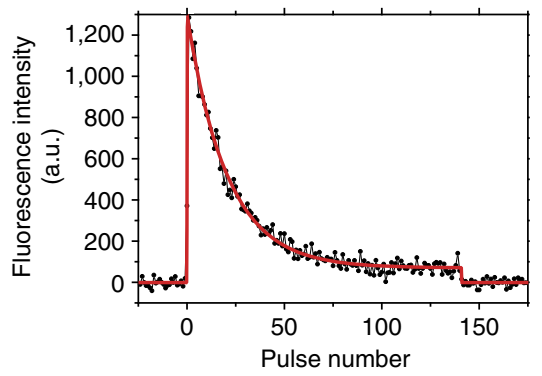

C

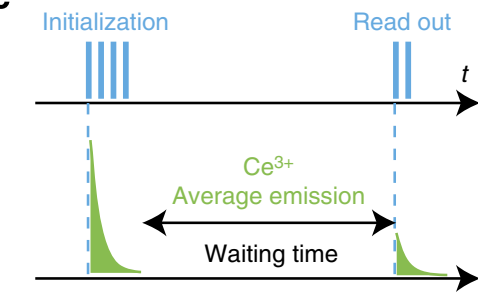

d

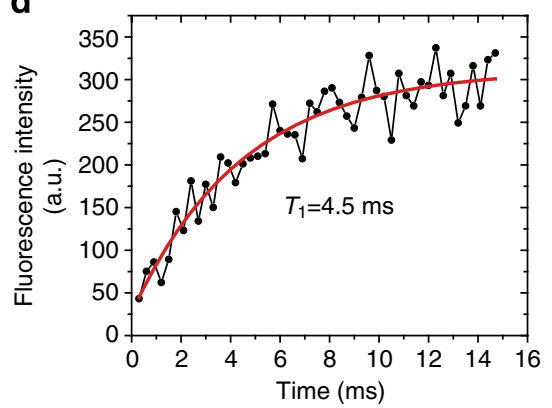

Figure 2 | Initialization and thermalization of $\mathbf{C e}^{\mathbf{3}+}$ spin. (a) Schematic diagram of the laser pulse sequence used to study the initialization dynamics. The time interval between the trains was chosen to be long enough for the spin population to thermalize. The photons accumulated after each pulse in the train are summed up and they represent one point on the initialization fluorescence curve. The detailed information on the excitation pulse sequence and on the fitting procedure is given in the Methods section. (b) Dependence of the fluorescence intensity on the pulse number during initialization process. (c) Laser pulse sequence used to measure spin relaxation time $T_{1}$. The initializing pulse train is long (140 pulses) to ensure spin polarization. The readout pulse train contains only five pulses. The time interval between initializing the spin and reading it out is varied. (d) Spin relaxation curve recorded by detecting the fluorescence during readout pulse train. In $\mathbf{b}$, $\mathbf{d}$ red curves are the fits to the observed signals.

evolution of the excited fluorescence was investigated (see Fig. 2a). As shown in Fig. 2b, right after the circularly polarized excitation is switched on, the emission intensity is maximal determined by thermalized populations of the ground-state spin sublevels. It rapidly drops because of optical pumping into the 'dark' spin state. The ratio of the fluorescence intensities in the beginning of the fluorescence decay and after the spin is polarized indicates $>97 \%$ fidelity of spin initialization. The fidelity of spin initialization not reaching the theoretically predicted ratio $396: 1$ is thought to be because of a slight ellipticity of the polarization caused by non-perfect $\lambda / 4$ waveplate and polarization distortion because of the SIL. Under linearly polarized excitation, cerium emission does not depend on time indicating the absence of optical pumping. The dependence of the emission on the ellipticity of the excitation is given in Supplementary Fig. 2.

The lifetime of the initialized spin state, $T_{1}$, was measured by introducing the second (readout) train of laser pulses having the same circular polarization and varying the time interval between initialization and readout (see Fig. 2c). The fluorescence recovery indicates thermalization of the spin states revealing in the spin relaxation time of $T_{1}=4.5 \mathrm{~ms}$ (see Fig. $2 \mathrm{~d}$ ).

Optical detection of $\mathrm{Ce}^{3+}$ spin resonance. The initialized spin can be manipulated by microwave radiation resonant with the spin transition of the ground $4 f(1)$ state. For that purpose, a microwave structure was created on the surface of the sample right next to the location of the SIL. This allowed us to record optically detected magnetic resonance $\left(O D M R^{17}\right)$ spectra of individual cerium ions. Once the frequency of microwaves matches that of the spin transition, the population from the $|4 f(1) \uparrow\rangle$ state is pumped back into $|4 f(1) \downarrow\rangle$ and the overall fluorescence yield increases. A typical ODMR spectrum of a single cerium ion is shown in Fig. 3a. Linewidths of ODMR spectra are in the range of $10-15 \mathrm{MHz}$ varying slightly for different cerium centres. The width of the ODMR line is determined by two factors: inhomogeneous broadening of the line because of random magnetic field produced by ${ }^{27} \mathrm{Al}$ nuclei and microwave power broadening. Since $\mathrm{Ce}^{3+}$ can occupy six magnetically inequivalent sites in YAG, in general, six distinct positions of ODMR peak are possible. A combination of measured ODMR frequency and known g-tensor of the ground-state $\mathrm{Ce}^{3+} \operatorname{spin}^{18}$ allowed us to determine the orientation of individual cerium sites. In our (110) oriented crystal, the ODMR resonances were at 640 and $1,310 \mathrm{MHz}$ and four nearly degenerate ones around 1,550 MHz. For further experiments, we were choosing the centres having their local $y$ axis (we adopted the axes assignment from the work ${ }^{19}$ ) oriented along the magnetic field and the excitation beam $(640 \mathrm{MHz})$ since for these centres the theoretical predictions for spin initialization fidelity is the best.

Coherent manipulation of $\mathrm{Ce}^{3+}$ spin. The first step towards coherent manipulation of cerium electron spin is detection of Rabi oscillations under strong microwave driving. Experimentally, the circularly polarized laser excitation was chopped such that only a few tens of laser pulses are used to initialize the spin in $|4 f(1) \uparrow\rangle$. After this, a microwave pulse at resonant frequency of variable duration was applied (see Fig. 3b). Finally, a short laser pulse train having identical circular polarization was applied to read out the population of the $|4 f(1) \downarrow\rangle$ spin state. The result of the measurement is shown in Fig. 3c. The frequency of Rabi oscillations has square-root dependence on microwave power. The decay time of oscillations is longer than determined by the inhomogeneous linewidth indicating partial locking of the spin by microwaves.

Dephasing of cerium spin was measured by decay of Hahn echo signal (a sequence of $\pi / 2-\tau-\pi-\tau-\pi / 2$ microwave pulses, see inset in Fig. 4a). The result yields a value of $T_{2}=2$ $\tau=240 \mathrm{~ns}$, where $\tau$ is the free evolution time between $\pi / 2$ and $\pi$ pulses. Fast decoherence in YAG can be explained by the presence 
a

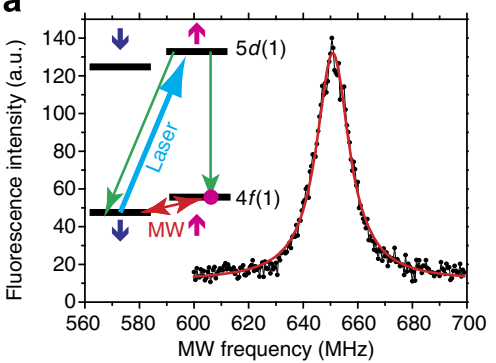

b

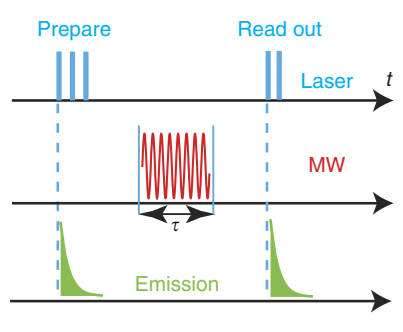

C

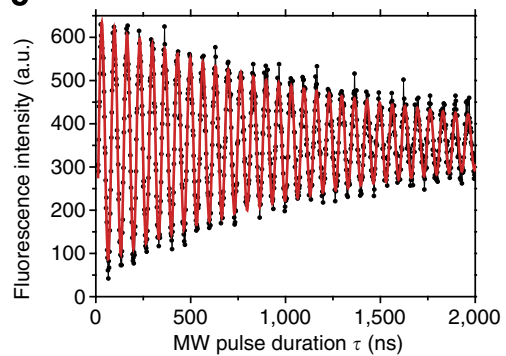

Figure 3 | Coherent manipulation of $\mathbf{C e}^{\mathbf{3}+}$ spin. (a) ODMR signal of a single $\mathrm{Ce}^{3+}$ ion as the frequency of microwaves (MW) is swept across the spin resonance. Inset shows level structure of the ground-state and the optically excited spin doublets. The red line is the Lorentzian fit to the observed signal. (b) Schematic diagram of Rabi measurements. (c) Spin Rabi oscillations of a single $\mathrm{Ce}^{3+}$ spin. The red line is the fit to the data. The observed signal was fitted with exponentially decaying cosine function with constant offset.

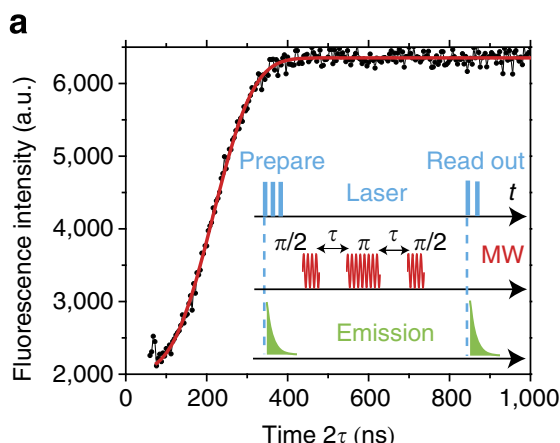

b

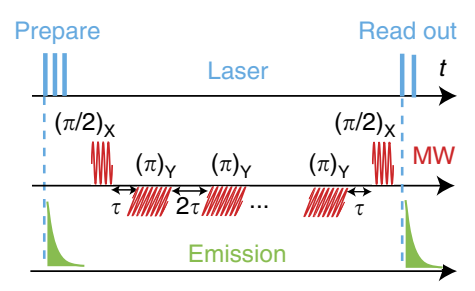

C

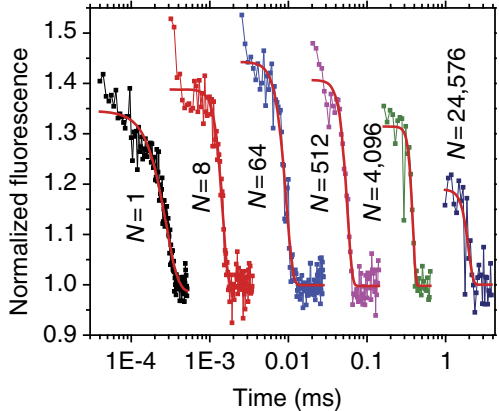

d

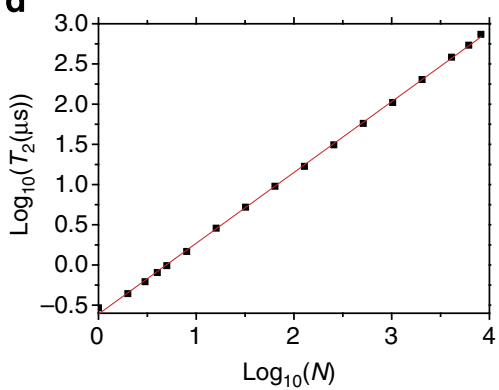

Figure 4 | Dynamic decoupling of $\mathbf{C e}^{\mathbf{3}+}$ spin from spin bath. (a) Spin-echo decay shows a decoherence time $T_{2}=240$ ns. The decay is non-exponential. The red curve represents Gaussian fit to the echo signal. (b) Schematic diagram of measuring the decoherence time with CPMG decoupling sequence. After applying the first $\pi / 2$ microwave pulse (X $\pi / 2$ pulse) and waiting for time $\tau$, one applies a sequence of $\pi$-microwave refocusing pulses separated by $2 \tau$ and phase-shifted from the $X \pi / 2$ pulse by $90^{\circ}(Y \pi$ pulses). Rotation along $y$-instead of $x$ plane on the Bloch sphere makes the sequence robust. (c) Results of CPMG measurements for several sequences. The number of pulses for each sequence is given for the corresponding decay curve. Stretched-exponential fits to the data are shown by red curves. (d) The dependence of the coherence lifetime on the length of CPMG sequence.

The red curve is the linear fit to the data points in log-log scale. The slope of 0.88 is closer to the linear dependence characteristic to nuclear spin bath rather than to $\mathrm{N}^{2 / 3}$ characteristic to the electron spin bath.

of the dense bath of ${ }^{27} \mathrm{Al}$ having spin of $I=5 / 2$ accompanied by large magnetic moment $\mu_{\mathrm{Al}}=3.64 \mu_{\mathrm{N}}$ with $\mu_{\mathrm{N}}$ being nuclear magneton. Fluctuating nuclear spins produce magnetic noise at the location of $\mathrm{Ce}^{3+}$ reducing coherence time. Theoretical analysis of the nuclear spin bath showed that the contribution of octahedrally coordinated ${ }^{27} \mathrm{Al}$ nuclei to decoherence is the largest (see Supplementary Methods).

Dynamic decoupling of $\mathrm{Ce}^{3+}$ spin from the nuclear bath. Dynamic decoupling exploiting sequences of $\pi$ microwave pulses allows for an enormous increase in decoherence time ${ }^{20}$. Decoherence is caused by the averaging over a phase factor $C(t)=\left\langle e^{i \varphi(t)}\right\rangle$ the $\mathrm{Ce}^{3+}$ spin acquires during free evolution. $\pi$ pulses applied with frequency $1 / \tau$ effectively eliminate noise spectral components $v_{\text {noise }}<1 / \tau$ and thus lead to lenghtening of coherence lifetime.

We used Carr-Purcell-Meiboom-Gill (CPMG) microwave decoupling sequence ${ }^{21,22}$ (see Fig. 4b). The maximum number of refocusing pulses applied was 24,576 . This allowed us to extend decoherence time up to $1.97 \mathrm{~ms}$. The measured CPMG-echo signals shown in Fig. 4c agree well with the ones simulated by noise spectrum analysis and cluster expansion method ${ }^{23}$ (see Supplementary Fig. 3). The dependence of the decoherence time on the number of CPMG pulses allows one to discriminate the influence of nuclear and electronic spin baths. The latter is composed of residual transition metal spin impurities such as $\mathrm{Cr}^{3+}$ and $\mathrm{Fe}^{3+}$. Electron and nuclear spin baths have 
significantly different noise spectral densities. The noise of the electron spin bath is best decribed by a Lorenzian-shaped spectrum characterized by a long spectral tail. This leads to a $N^{2 / 3}$ depencence of the coherence time ${ }^{24,25}$. On the other hand, nuclear spin noise is characterized by a much harder cutoff leading to a linear dependence of $T_{2}$ on $N$. The experimentally measured dependence shown inFig. $4 \mathrm{~d}$ is $T_{2} \propto N^{0.88}$ indicating that both nuclear and electron spin baths lead to decoherence with former contribution prevailing. Stretched-exponential fits $\left(\exp \left(-\left(t / T_{2}\right)^{k}\right)\right)$ to CPMG-echo signals result in exponentials $k$ between 6 and 12 confirming the existence of hard cutoff of the nuclear spin noise spectrum as described in Supplementary Methods. These results suggest that changing the host material to a low nuclear spin one-that is, yttrium orthosilicate in which rare-earth ions show the longest coherence and population lifetimes $5,26,27$, should greately increase coherence lifetime in our single spin experiments. Changing the host material has almost no effect on the atomic-like polarization selection rules of $\mathrm{Ce}^{3+}$ crucial for initializing its spin state. Preliminary measurements indeed show extended coherence lifetime in the excited $5 d$ state in yttrium orthosilicate compared with YAG (see Supplementary Fig. 4).

\section{Discussion}

An important asset of YAG and similar materials is that they are suitable for integrated photonic circuit fabrication, for example, in the form of thin films on low refractive index substrates (quartz) ${ }^{28,29}$. Subsequent FIB milling would allow for creating onchip photonic circuits with low attenuation $\left(1.5 \mathrm{~dB} \mathrm{~cm}^{-1}\right.$ (ref. 28)) for single photons, which would include waveguides, cavities and so on. This would result in technologically simple realization of an on-chip integration of cerium spin qubits and, simultaneously, in obtaining access to longer-living isolated nuclear spins magnetically coupled the electron spin of $\mathrm{Ce}^{3+}$.

\section{Methods}

Experimental setup. Optical studies of single $\mathrm{Ce}^{3+}$ ions were performed in a home-built confocal microscope in which the crystal was mounted on a cold finger of a helium flow cryostat. An optical access was arranged through a window. The microscope objective lens of numerical apperture 0.95 was mounted inside the cryostat on a piezo nano-positioner. A toroidal permanent magnet was fixed on the objective lens to provide magnetic field parallel to the propagation direction of the excitation laser beam. The fluorescence of cerium ions was collected by the same objective lens and detected with a single photon counting avalanche photodiode (APD). To perform photon-correlation measurements, the fluorescence signal was split by a $50 / 50$ beamsplitter and detected by two separate APDs. The signals of the two APDs were analysed by Becker and Hickl time-correlated single-photon counter card. In order to measure the emission spectra, the fluorescence was deflected by a mirror mounted on a flip mount on a grating spectrometer equipped with a cooled CCD camera. For measurements involving microwaves, a high-power MW amplifier (50 dB amplification, $30 \mathrm{~W}$ maximum output power) was used. The frequency of microwaves was swept by a software-controlled MW synthesizer. The microwaves were supplied to the sample by a single copper wire positioned next to the SIL. The wire was lithographically defined on the surface of the crystal and electroplated. The cross-section of the wire amounted to $20 \mu \mathrm{m}$ wide and $10 \mu \mathrm{m}$ high with its length being several hundred microns. The wire was electrically contacted to the microwave source on one side and to the $50-\Omega$-terminated drain on the other. No measures were taken to ensure impedance matching. However, the measured MW loss in the wire amounted to only $6 \mathrm{~dB}$.

Sample preparation. In order to improve fluorescence collection efficiency, a solid immersion microlens was fabricated directly on the surface of the sample by FIB milling. The lens had a shape of half-a-sphere with the radius of $5 \mu \mathrm{m}$. In order to perform spin manipulations with microwaves, a copper microwave structure was created next to the location of the SIL by lithographic means. Even though the microwave structure was not impedance-matched to the outer MW network, the measured overall MW loss amounted to only $6 \mathrm{~dB}$.

Details on initialization of $\mathbf{C e}^{\mathbf{3}+}$ spin state. The pumping dynamics of the ground-state spin state was revealed in the following way. A finite train of circularly polarized laser pulses (the duration of the pulse is $100 \mathrm{fs}$ ) was shot on the single
$\mathrm{Ce}^{3+}$ centre. The train contained 140 pulses with the repetition rate $2.5 \mathrm{MHz}$. The average laser power within the train was $50 \mu \mathrm{W}$ at the entrance aperture of the microscope objective lens. After each pulse train, the cerium ion spin was allowed to relax to thermal equillibrium for $50 \mathrm{~ms}$. After that, the excitation of the ion with the finite pulse train was repeated and the signals obtained for each pulse train were averaged. The resulting signal was fitted with an exponential decay function multiplied by two step-functions (step-up and step-down) in order to simulate the beginning and the end of the excitation pulse train.

Implantation of cerium ions. YAG crystals were implanted in EIKO FIB with accelerating voltage $100 \mathrm{kV}$. Ions were extracted from the liquid metal ion source Ce$\mathrm{Si}-\mathrm{Au}$. Ions were separated with built-in Wien filter so that $\mathrm{Ce}^{3+}$ were implanted with the energy of $300 \mathrm{keV}$. With the simulations performed in the SRIM software, we obtain penetration depth of $62 \mathrm{~nm}$ with straggle of $18 \mathrm{~nm}$. Crystal was implanted with pattern of dots with fluences of $10^{11} \mathrm{~cm}^{-2}$ and $2 \times 10^{12} \mathrm{~cm}^{-2}$. This corresponds to $\sim 10^{3}$ and $2 \times 10^{4}$ ions implanted per spot. In order to activate the implanted ions, thermal annealing was performed in argon atmosphere at $1,100^{\circ} \mathrm{C}$ for $1 \mathrm{~h}$. The activation yield was estimated by comparing the brightness of the implanted spots with the brightness of a single native cerium ion.

\section{References}

1. de Riedmatten, H., Afzelius, M., Staudt, M. U., Simon, C. \& Gisin, N. A solidstate lightmatter interface at the single-photon level. Nature 456, 773-777 (2008).

2. Clausen, C. et al. Quantum storage of photonic entanglement in a crystal. Nature 469, 508-511 (2011).

3. Saglamyurek, E. et al. Broadband waveguide quantum memory for entangled photons. Nature 469, 512-515 (2011).

4. Longdell, J. J., Fraval, E., Sellars, M. J. \& Manson, N. B. Stopped light with storage times greater than one second using electromagnetically induced transparency in a solid. Phys. Rev. Lett. 95, 063601 (2005).

5. Heinze, G., Hubrich, C. \& Halfmann, T. Stopped light and image storage by electromagnetically induced transparency up to the regime of one minute. Phys. Rev. Lett. 111, 033601 (2013).

6. Vivoli, V. C., Sangouard, N., Afzelius, M. \& Gisin, N. High-bandwidth quantum memory protocol for storing single photons in rare-earth doped crystals. New J. Phys. 15, 095012 (2013).

7. Timoney, N., Usmani, I., Jobez, P., Afzelius, M. \& Gisin, N. Single-photon-level optical storage in a solid-state spin-wave memory. Phys. Rev. A 88, 022324 (2013).

8. Sangouard, N., Simon, C., de Riedmatten, H. \& Gisin, N. Quantum repeaters based on atomic ensembles and linear optics. Rev. Mod. Phys. 83, 33-80 (2011).

9. Knill, E., Laflamme, R. \& Milburn, G. J. A scheme for efficient quantum computation with linear optics. Nature 409, 46-52 (2001).

10. Longdell, J. J., Sellars, M. J. \& Manson, N. B. Demonstration of conditional quantum phase shift between ions in a solid. Phys. Rev. Lett. 93, 130503 (2004)

11. Kolesov, R. et al. Optical detection of a single rare-earth ion in a crystal. Nat. Commun. 3, 1029 (2012).

12. Yin, C. et al. Optical addressing of an individual erbium ion in silicon. Nature 497, 91-94 (2013).

13. Kolesov, R. et al. Mapping spin coherence of a single rare-earth ion in a crystal onto a single photon polarization state. Phys. Rev. Lett. 111, 120502 (2013).

14. Utikal, T. et al. Spectroscopic detection and state preparation of a single praseodymium ion in a crystal. Nat. Commun. 5, 3627 (2014).

15. Robbins, D. J. J. Electrochem. Soc. 126, 1550-1555 (1979).

16. Varney, C. R., Mackay, D. T., Reda, S. M. \& Selim, F. A. On the optical properties of undoped and rare-earth-doped yttrium aluminium garnet single crystals. J. Phys. D Appl. Phys. 45, 015103 (2012).

17. Morigaki, K. Optically detected magnetic resonance. Semiconduct. Semimet. 21C, 155-191 (1984).

18. Lewis, H. R. Paramagnetic resonance of $\mathrm{Ce}^{3+}$ in yttrium aluminum garnet. J. Appl. Phys. 37, 739-741 (1966).

19. van der Ziel, J. P., Sturge, M. D. \& van Uitert, L. G. Optical detection of site selectivity for rare-earth ions in flux-grown yttrium aluminum garnet. Phys. Rev. Lett. 27, 508-511 (1971).

20. Viola, L. \& Lloyd, S. Dynamical suppression of decoherence in two-state quantum systems. Phys. Rev. A 58, 2733-2744 (1998).

21. Carr, H. Y. \& Purcell, E. M. Effects of diffusion on free precession in nuclear magnetic resonance experiments. Phys. Rev. 94, 630-638 (1954).

22. Meiboom, S. \& Gill, D. Modified spin echo method for measuring nuclear relaxation times. Rev. Sci. Instrum. 29, 688-691 (1958).

23. Yang, W. \& Liu, R.-B. Quantum many-body theory of qubit decoherence in a nite-size spin bath. Phys. Rev. B 78, 085315 (2008).

24. de Lange, G., Wang, Z. H., Risté, D., Dobrovitski, V. V. \& Hanson, R. Universal dynamical decoupling of a single solid-state spin from a spin bath. Science 330, 60-63 (2010)

25. Medford, J. et al. Scaling of dynamical decoupling for spin qubits. Phys. Rev. Lett. 108, 086802 (2012). 
26. Yan, Y. et al. Measurement of linewidths and permanent electric dipole moment change of the Ce $4 f-5 d$ transition in $\mathrm{Y}_{2} \mathrm{SiO}_{5}$ for qubit readout scheme in rare-earth ion based quantum computing. Phys. Rev. B 87, 184205 (2013).

27. Könz, F. et al. Temperature and concentration dependence of optical dephasing, spectral-hole lifetime, and anisotropic absorption in $\mathrm{Eu}^{3+}: \mathrm{Y}_{2} \mathrm{SiO}_{5}$. Phys. Rev. B 68, 085109 (2003).

28. Wu, Y.-C., Parola, S., Marty, O., Villanueva-Ibanez, M. \& Mugnier, J. Structural characterizations and waveguiding properties of YAG thin films obtained by different solgel processes. Opt. Mater. 27, 1471-1479 (2005).

29. Choe, J. Y. Luminescence and compositional analysis os $\mathrm{Y}_{3} \mathrm{Al}_{5} \mathrm{O}_{12}$ :Ce films fabricated by pulsed-laser deposition. Mat. Res. Innovat. 6, 238-241 (2002).

\section{Acknowledgements}

The work was financially supported by EU project SQUTEC, Volkswagen Foundation, SFB TR 21, the Max Planck Society, BMBF-QUIMP 16BQ1062, and DFH/UFA CDFA05-06 Nice-Bochum.

\section{Author contributions}

P.S. and K.X. contributed equally to this work; P.S., K.X., R.R., M.J., N.K., A.D.W. and R.K. performed the experiments; P.S., K.X. and R.K. designed the experiments; P.S., K.X. and R.K. analysed the data; N.Z., N.Y. and C.D. performed theoretical calculations; R.K., N.Z. and J.W. wrote the paper; J.W. supervised the project.

\section{Additional information}

Supplementary Information accompanies this paper at http://www.nature.com/ naturecommunications

Competing financial interests: The authors declare no competing financial interests.

Reprints and permission information is available online at http://npg.nature.com/ reprintsandpermissions/

How to cite this article: Siyushev, P. et al. Coherent properties of single rare-earth spin qubits. Nat. Commun. 5:3895 doi: 10.1038/ncomms4895 (2014). 